\title{
Further Results on Output Tracking Problem of Uncertain Nonlinear Systems with High-Order Nonlinearities
}

\author{
Keylan Alimhan ${ }^{1,2}$, Naohisa Otsuka ${ }^{2,}$ Maksat N. Kalimoldayev ${ }^{3}$, \\ Abilmajin A. Adamov ${ }^{1}$ \\ ${ }^{1}$ L.N. Gumilyov Eurasian National University, \\ Astana, Kazakhstan \\ keylan@live.jp; adam1955@mail.ru \\ ${ }^{2}$ Division of Science, School of Science and Engineering, \\ Tokyo Denki University, \\ Hatayama-Machi, Hiki-Gun, Saitama 350-0394, Japan \\ otsuka@mail.dendai.ac.jp \\ ${ }^{3}$ Institute of Information and Computing Technologies, \\ Almaty, Kazakhstan \\ mnk@ipic.kz.
}

\begin{abstract}
This paper considers the global practical tracking problem by state feedback for a class of high-order non-linear systems with more general uncertainties, to which the existing control methods are inapplicable. We successfully propose a new tracking control design scheme for the system studied by introducing sign function and necessarily modifying the method of adding a power integrator. It is shown that the designed controller guarantees that all states of the resulting closed-loop system are globally bounded and the tracking error remains prescribed arbitrarily small after a finite time.
\end{abstract}

Keywords: uncertain nonlinear systems, practical output tracking, sign function, state feedback

\section{Introduction}

The output tracking problem is one of most important subjects in control theory and its applications, and it has been extensively studied for the last three decades. Its basic problem is to design a feedback control law making the controlled output track a given reference signal as much as possible. The output tracking in the usual case is in the sense of "asymptotic" where the tracking error converges to zero as time goes to the infinity, and this asymptotic tracking problem for time-invariant linear systems was completely solved about thirty years ago.

The corresponding problem for nonlinear systems was also carried out by a number of researchers at least over the past twenty years $[1,2]$, etc.

However, in the case of inherently nonlinear systems where the linearized systems may not be stabilizable and/or detectable, the tracking problem and even the stabilization problem become much more complicated and difficult to solve.

Thus, to overcome this difficulty, a new concept called practical output tracking for tracking problem has been introduced and various results in the framework of the new concept have been reported see [3-9], as well as the references therein. 
This paper deals with the practical output tracking problem with a state feedback for a class of high-order nonlinear systems having the following form:

$$
\begin{aligned}
\dot{z}_{1} & =z_{2}^{p_{1}}+\phi_{1}(t, z, u) \\
\dot{z}_{2} & =z_{3}^{p_{2}}+\phi_{2}(t, z, u) \\
& \vdots \\
\dot{z}_{n-1} & =z_{n}^{p_{n-1}}+\phi_{n-1}(t, z, u), \\
\dot{z}_{n} & =u+\phi_{n}(t, z, u), \\
y & =z_{1},
\end{aligned}
$$

where $z=\left(z_{1}, \ldots, z_{n}\right)^{\mathrm{T}} \in R^{n}$ and $u \in R$ are the system state and the control input, respectively. For $i=1, \ldots, n, \phi_{i}(t, z, u)$ are unknown continuous functions and $p_{i} \in R_{\text {odd }}^{\geq 1}:=\left\{p / q \in R^{+}: p\right.$ and $q$ are odd integers, $\left.p \geq q\right\}(i=1, \ldots, n-1)$ are said to be the high orders of the system, with $p_{n}$ obviously equal to one (which is not a limitation since we can easily set $v:=u^{p_{n}}$ in the case of non-unity $p_{n}$ ).

We first introduce definition of the practical output tracking problem.

Consider the system (1) and assume that the reference signal $y_{r}(t)$ be a time-varying $C^{1}$ bounded on $[0, \infty)$. Then, the global practical output tracking problem by a state controller is formulated as follows: For any given real number $\varepsilon>0$, design a continuous controller having the following structure

$$
u=u\left(z, y_{r}(t)\right),
$$

such that

(i) all the states of the closed-loop system (1) and (2) are well-defined on $[0, \infty)$ and globally bounded;

(ii) the global practical output tracking is achieved, that is, for every $z(0) \in R^{n}$ there is a finite time $T:=T_{(\varepsilon, z(0))}>0$, such that the output $y(t)$ of the closed loop system (1) with (2) satisfies

$$
\left|y(t)-y_{r}(t)\right|=\left|z_{1}(t)-y_{r}(t)\right|<\varepsilon, \quad \forall t \geq T>0 .
$$

The problem of output tracking control of nonlinear systems is one of the most important and challenging problems in the field of nonlinear control and lots of efforts have been made during the last decades, see [1-11], as well as the references therein. With the help of the nonlinear output regulator theory [1], [2] and the method of adding a power integrator [12-14], series of research results have been obtained [3-5]. For details, in [3], practical output tracking via smooth state feedback for nonlinear systems was considered. Further, in [7-9], the practical output feedback tracking problem was also investigated for a class of nonlinear systems with higher-order growing unmeasurable states, extending the results on stabilization in [15-18].

In [7-9], the following condition on the uncertain term $\phi_{i}(\cdot)$ is assumed:

$$
\left|\phi_{i}(t, z, u)\right| \leq C\left(\left|z_{1}\right|^{\left(r_{i}+\tau\right) / r_{1}}+\cdots+\left|z_{i}\right|^{\left(r_{i}+\tau\right) / r_{i}}\right)+C
$$

where $C>0, \tau>0$ or $-2 / p_{1} p_{2} \cdots p_{n-1}(2 n+1)<\tau<0$ are constants and $r_{i}$ 's are defined as $r_{1}=1, r_{i+1} p_{i}=r_{i}+\tau>0, \quad i=1, \ldots, n$. However, (4) needs the condition of $\tau=l / m$ with $l$ being an even integer and $m$ being an odd integer, which results in $\left(r_{i}+\tau\right) / r_{j}$ in (4) being always a ratio of odd integers. Naturally, an interesting problem may be proposed: 
(a) Is it possible to relax the assumption on $\tau$ in (4)? (b) Under the weaker assumption, can one design an output tracking controller?

In this paper, by introducing the sign function approach, and overcoming several troublesome obstacles in the design and analysis procedure, we focus on solving the above problem under the assumption of the restriction on $\tau$ being relaxed to any real number.

\section{Mathematical Preliminaries}

At first, we give the following notations which will be used in this study.

Notations: $R^{+}$denotes the set of all the nonnegative real numbers and $R^{n}$ denotes the real $n$-dimensional space. For any vector $x=\left(x_{1}, \ldots, x_{n}\right)^{T} \in R^{n}$, denote

$$
\bar{x}_{i}:=\left(x_{1}, \ldots, x_{i}\right)^{T} \in R^{i}, i=1, \ldots, n,\|x\|:=\sqrt{\sum_{i=1}^{n} x_{i}^{2}} .
$$

A sign function $\operatorname{sgn}(x)$ is defined as: $\operatorname{sgn}(x)=1$ if $x>0, \operatorname{sgn}(x)=0$ if $x=0$, and $\operatorname{sgn}(x)=-1$ if $x<0$.

In order to solve the global practical output tracking problem, we made the following assumption:

Assumption1 For $i=1, \ldots, n$, there are smooth functions $\psi_{i j}\left(z_{1}, \ldots, z_{i}\right), j=1,2$ and $\tau \in\left(-1 / \sum_{l=1}^{n} p_{1} \cdots p_{l-1}, 0\right)$ such that

$$
\left|\phi_{i}(t, z, u)\right| \leq \psi_{i 1}\left(z_{1}, \ldots, z_{i}\right)\left(\left|z_{1}\right|^{\left(r_{i}+\tau\right) / r_{1}}+\cdots+\left|z_{i}\right|^{\left(r_{i}+\tau\right) / r_{i}}\right)+\psi_{i 2}\left(z_{1}, \ldots, z_{i}\right)
$$

where $r_{i}^{\prime}$ s defined as

$$
r_{1}=1, \quad r_{i+1} p_{i}=r_{i}+\tau>0, \quad i=1, \ldots, n .
$$

Now, we introduce six technical lemmas which will play an important role and be frequently used in the later control design.

Lemma1[3]. For any real numbers $x \geq 0, y>0$ and $m \geq 1$, the following inequality holds:

$$
x \leq y+(x / m)^{m}((m-1) / y)^{m-1} .
$$

Lemma2[19]. For all $x, y \in R$ and a constant $p \geq 1$ the following inequalities holds:

(i) $|x+y|^{p} \leq 2^{p-1}\left|x^{p}+y^{p}\right|$,

$$
(|x|+|y|)^{1 / p} \leq|x|^{1 / p}+|y|^{1 / p} \leq 2^{(p-1) / p}(|x|+|y|)^{1 / p}
$$

If $p \in R_{o d d}^{\geq 1}$, then

(ii) $|x-y|^{p} \leq 2^{p-1}\left|x^{p}-y^{p}\right|$,

$$
\left|x^{1 / p}-y^{1 / p}\right| \leq 2^{(p-1) / p}|x-y|^{1 / p} .
$$


Lemma3[19]. Let $c, d$ be positive constants. Then, for any real-valued function $\gamma(x, y)>0$, the following inequality holds:

$$
|x|^{c}|y|^{d} \leq \frac{c}{c+d} \gamma(x, y)|x|^{c+d}+\frac{d}{c+d} \gamma^{-c / d}(x, y)|y|^{c+d} .
$$

Lemma4[20]. For $x, y \in R$ and $0<p \leq 1$ the following inequality holds

$$
(|x|+|y|)^{p} \leq|x|^{p}+|y|^{p} .
$$

When $p=a / b \leq 1$, where $a>0$ and $b>0$ are $o d d$ integers

$$
\left|x^{p}+y^{p}\right| \leq 2^{1-p}|x+y|^{p} .
$$

Lemma5[21]. If $p=a / b \in R_{\text {odd }}^{\geq 1}$ with $a \geq b \geq 1$ being some real numbers, then for any $x, y \in R$

$$
\left|x^{p}-y^{p}\right| \leq\left. 2^{1-1 / b}|\operatorname{sgn}(x)| x\right|^{a}-\left.\operatorname{sgn}(y)|y|^{a}\right|^{1 / b}
$$

Lemma6[6]. If $f:[a, b] \rightarrow R(a \leq b)$ is monotone continuous and satisfies $f(a)=0$, then

$$
\left|\int_{a}^{b} f(x) d x\right| \leq|f(b)| \cdot|b-a|
$$

\section{Construction of Tracking Control}

In this section, we will present a recursive design approach to construct the tracking control for system (1). For simplicity, we denote $\operatorname{sgn}(x)|x|^{\alpha}:=[x]^{\alpha}$ for any $\alpha \in R^{+}$and $x \in R$.

The following theorem is the main result of this paper.

Theorem 1. Let $y_{r}(t)$ be a reference signal whose derivative $\dot{y}_{r}(t)$ is also bounded. Then, under Assumption1, the global practical output tracking problem of the system (1) is solvable by a continuous state feedback controller of the form (2).

Proof: The inductive proof relies on the simultaneous construction of a $C^{1}$ Lyapunov function which is positive define and proper, as well as a homogeneous-like controller at each iteration.

Let $x_{1}=z_{1}-y_{r}$ and given $x_{i}=z_{i}, i=2, \ldots, n$. Then, we have

$$
\begin{aligned}
\dot{x}_{1} & =x_{2}^{p_{1}}+\phi_{1}\left(t, x_{1}+y_{r}, x_{2}, \ldots, x_{n}, u\right)-\dot{y}_{r}(t), \\
\dot{x}_{2} & =x_{3}^{p_{2}}+\phi_{2}\left(t, x_{1}+y_{r}, x_{2}, \ldots, x_{n}, u\right), \\
& \vdots \\
\dot{x}_{n-1} & =x_{n}^{p_{n-1}}+\phi_{n-1}\left(t, x_{1}+y_{r}, x_{2}, \ldots, x_{n}, u\right), \\
\dot{x}_{n} & =u+\phi_{n}\left(t, x_{1}+y_{r}, x_{2}, \ldots, x_{n}, u\right), \\
y & =x_{1}+y_{r} .
\end{aligned}
$$

Initial Step. Let $\xi_{1}=\left[x_{1}\right]$ and construct the Lyapunov function as

$$
V_{1}\left(x_{1}\right)=W_{1}\left(\bar{x}_{1}\right)=\int_{0}^{x_{1}} s^{2-r_{2} p_{1}} d s,
$$


where

$$
W_{1}\left(\bar{x}_{1}\right)=\int_{x_{1}^{*}}^{x_{1}^{*}}\left[[s]^{1 / r_{1}}-\left[x_{1}^{*}\right]^{1 / r_{1}}\right]^{2-r_{2} p_{1}} d s
$$

and $x_{1}^{*} \equiv 0$ for convenience. Note that $V_{1}$ is $C^{1}$, positive definite and proper.

A direct calculation gives

$$
\dot{V}_{1}\left(x_{1}\right)=x_{1}^{2-r_{2} p_{1}}\left[x_{2}^{p_{1}}+\phi_{1}\left(t, x_{1}+y_{r}, x_{2}, \ldots, x_{n}, u\right)-\dot{y}_{r}(t)\right] .
$$

Since $y_{r}(t)$ and $\dot{y}_{r}(t)$ are bounded and by Assumption1 and Lemmas1-6, it can be shown that there is a smooth functions $\tilde{\psi}_{1}\left(x_{1}\right)$ such that

$$
\begin{aligned}
\left|\phi_{1}\left(t, x+y_{r}, x_{2} \ldots, x_{n}, u\right)-\dot{y}_{r}\right| & \leq \psi_{11}\left(x_{1}+y_{r}\right)\left|x_{1}+y_{r}\right|^{\left(r_{1}+\tau\right) / r_{1}}+\psi_{12}\left(x_{1}+y_{r}\right)+M \\
& \leq \tilde{\psi}_{11}\left(x_{1}\right)\left|x_{1}\right|^{\left(r_{1}+\tau\right) / r_{1}}+\tilde{\psi}_{11}\left(x_{1}\right) M^{\left(r_{1}+\tau\right) / r_{1}}+\tilde{\psi}_{12}\left(x_{1}\right)+M \\
& \leq \tilde{\psi}_{1}\left(x_{1}\right)\left|x_{1}\right|^{\left(r_{1}+\tau\right) / r_{1}}+\kappa_{1}\left(x_{1}\right)
\end{aligned}
$$

satisfying

$$
\dot{V}_{1}\left(x_{1}\right) \leq x_{1}^{2-r_{2} p_{1}} x_{2}^{* p_{1}}+x_{1}^{2-r_{2} p_{1}}\left(x_{2}^{p_{1}}-x_{2}^{* p_{1}}\right)+x_{1}^{2}\left(\tilde{\psi}_{11}\left(x_{1}\right)+\alpha_{1}\left(x_{1}\right)\right)+\delta,
$$

where $\delta>0$ is any real constant,

$$
\alpha_{1}\left(x_{1}\right)=\left(\frac{\left(2-r_{2} p_{1}\right) \kappa_{1}\left(x_{1}\right)}{2}\right)^{2 /\left(2-r_{2} p_{1}\right)}\left(\frac{r_{2} p_{1}}{\left(2-r_{2} p_{1}\right) \delta}\right)^{r_{2} p_{1} /\left(2-r_{2} p_{1}\right)}
$$

and

$$
\kappa_{1}\left(x_{1}\right) \geq \tilde{\psi}_{11}\left(x_{1}\right) M^{\left(r_{1}+\tau\right) / r_{1}}+\tilde{\psi}_{12}\left(x_{1}\right)+M \text {. }
$$

Define a smooth positive function $\tilde{\kappa}_{1}\left(x_{1}\right)$ such that $\tilde{\kappa}_{1}\left(x_{1}\right) \geq \alpha_{1}\left(x_{1}\right)+\kappa_{1}\left(x_{1}\right)$. Then, we have

$$
\dot{V}_{1}\left(x_{1}\right) \leq\left[\xi_{1}\right]^{2-r_{2} p_{1}} x_{2}^{* p_{1}}+\left[\xi_{1}\right]^{2-r_{2} p_{1}}\left(x_{2}^{p_{1}}-x_{2}^{*} p_{1}\right)+x_{1}^{2} \tilde{\kappa}_{1}\left(x_{1}\right)+\delta .
$$

If we take the virtual controller $x_{2}^{*}$ as

$$
x_{2}^{*}=-\beta_{1}^{r_{2}}\left(x_{1}\right) x_{1}^{r_{2}}=-\beta_{1}^{r_{2}}\left(x_{1}\right)\left[\xi_{1}\right]^{r_{2}},
$$

where $\beta_{1}\left(x_{1}\right) \geq\left(n+\tilde{\kappa}_{1}\left(x_{1}\right)\right)^{1 / r_{2} p_{1}}$, then it follows that

$$
\dot{V}_{1}\left(x_{1}\right) \leq-n x_{1}^{2}+\left[\xi_{1}\right]^{2-r_{2} p_{1}}\left(x_{2}^{p_{1}}-x_{2}^{* p_{1}}\right)+\delta .
$$

Inductive Step. Suppose at the (k-1)-th step, there is a $C^{1}$, positive definite and proper Lyapunov function $V_{k-1}\left(x_{1}, \ldots, x_{k-1}\right)$, which is positive definite and proper, and a set of virtual controllers $x_{1}^{*}, \ldots, x_{k}^{*}$ defined by

$$
\begin{array}{rlrl}
x_{1}^{*} & =0, & \xi_{1} & =\left[x_{1}\right]^{1 / r_{1}}-\left[x_{1}^{*}\right]^{1 / r_{1}}, \\
x_{2}^{*} & =-\beta_{1}^{r_{2}}\left(\bar{x}_{1}\right)\left[\xi_{1}\right]^{r_{2}}, & & \xi_{2}=\left[x_{2}\right]^{1 / r_{2}}-\left[x_{2}^{*}\right]^{1 / r_{2}}, \\
\vdots & & \\
x_{k}^{*}=-\beta_{k-1}^{r_{k}}\left(\bar{x}_{k-1}\right)\left[\xi_{k-1}\right]^{r_{k}}, & \xi_{k}=\left[x_{k}\right]^{1 / r_{k}}-\left[x_{k}^{*}\right]^{1 / r_{k}},
\end{array}
$$


with $\beta_{i}\left(\bar{x}_{i}\right), 1 \leq i \leq k-1$, being smooth positive functions, such that

$$
\begin{aligned}
\dot{V}_{k-1}\left(\bar{x}_{k-1}\right) \leq & -(n-k+2)\left(\xi_{1}^{2}+\cdots+\xi_{k-1}^{2}\right) \\
& +\left[\xi_{k-1}\right]^{2-r_{k} p_{k-1}}\left(x_{k}^{p_{k-1}}-x_{k}^{*} p_{k-1}\right)+(k-1) \delta .
\end{aligned}
$$

We claim that (12) also holds at Step $k$. To prove this claim, we choose the following Lyapunov function

$$
V_{k}\left(\bar{x}_{k}\right)=V_{k-1}\left(\bar{x}_{k-1}\right)+W_{k}\left(\bar{x}_{k}\right)
$$

where

$$
W_{k}\left(\bar{x}_{k}\right)=\int_{x_{k}^{*}}^{x_{k}}\left[[s]^{1 / r_{k}}-\left[x_{k}^{*}\right]^{1 / r_{k}}\right]^{2-r_{k+1} p_{k}} d s .
$$

Noting that $2-r_{k+1} p_{k} \geq 1$ and using a similar method as in [21], $V_{k}(\cdot)$ can be shown to be $C^{1}$, proper and positive definite. Moreover, we can obtain

$$
\begin{gathered}
\frac{\partial W_{k}}{\partial x_{i}}=-\left(2-r_{k+1} p_{k}\right) \int_{x_{k}^{*}}^{x_{k}}\left[[s]^{1 / r_{k}}-\left[x_{k}^{*}\right]^{1 / r_{k}}\right]^{1-r_{k+1} p_{k}} d s \frac{\partial\left(\left[x_{k}^{*}\right]^{1 / r_{k}}\right)}{\partial x_{i}}, \\
\frac{\partial W_{k}}{\partial x_{k}}=\left[[x]^{1 / r_{k}}-\left[x_{k}^{*}\right]^{1 / r_{k}}\right]^{2-r_{k+1} p_{k}}=\left[\xi_{k}\right]^{2-r_{k+1} p_{k}}
\end{gathered}
$$

where $i=1, \ldots, k-1$, and there is a known constant $L>0$, such that

$$
W_{k} \geq L\left(x_{k}-x_{k}^{*}\right)^{2-r_{k+1} p_{k}} .
$$

Using (12)-(15), it follows that

$$
\begin{aligned}
\dot{V}_{k}\left(\bar{x}_{k}\right) \leq-(n-k+2) & \sum_{i=1}^{k-1} \xi_{i}^{2}+\left[\xi_{k-1}\right]^{2-r_{k} p_{k-1}}\left(x_{k}^{p_{k-1}}-x_{k}^{*} p_{k-1}\right) \\
+ & (k-1) \delta+\left[\xi_{k}\right]^{2-r_{k+1} p_{k}}\left(x_{k+1}^{* p_{k}}+\psi_{k}(\cdot)\right) \\
& +\sum_{i=1}^{k-1} \frac{\partial W_{k}}{\partial x_{i}}\left(x_{i+1}^{p_{i}}+\psi_{i}(\cdot)\right)+\left[\xi_{k}\right]^{2-r_{k+1} p_{k}}\left(x_{k+1}^{p_{k}}-x_{k+1}^{* p_{k}}\right)
\end{aligned}
$$

for a virtual controller $x_{k+1}^{* p_{k}}$ to be determined later. In order to proceed further, an appropriate bounding estimate should be given for the last three terms on the right hand side of inequality (17). This is accomplished in the following three facts whose technical proofs are given in the appendix.

Fact 1: There exists a positive constant $a_{k}$ such that

$$
\left[\xi_{k-1}\right]^{2-r_{k} p_{k-1}}\left(x_{k}^{p_{k-1}}-x_{k}^{*} p_{k-1}\right) \leq \frac{1}{3} \xi_{k-1}^{2}+a_{k 1} \xi_{k}^{2} .
$$

Fact 2: There exists a nonnegative smooth function $b_{k}\left(\bar{x}_{k}\right)$ such that

$$
\left[\xi_{k}\right]^{2-r_{k+1} p_{k}} \psi_{k}\left(t, x_{1}+y_{r}, x_{2}, \ldots, x_{k}, u\right) \leq \frac{1}{3} \sum_{i=1}^{k-1} \xi_{i}^{2}+b_{k}\left(\bar{x}_{k}\right) \xi_{k}^{2}+\frac{1}{2} \delta .
$$

Fact 3: There exists a nonnegative smooth functions $c_{k}\left(\bar{x}_{k}\right)$ such that 


$$
\sum_{i=1}^{k-1} \frac{\partial W_{k}}{\partial x_{i}}\left(x_{i+1}^{p_{i}}+\psi_{i}(\cdot)\right) \leq \frac{1}{3} \sum_{i=1}^{k-1} \xi_{i}^{2}+c_{k}\left(\bar{x}_{k}\right) \xi_{k}^{2}+\frac{1}{2} \delta .
$$

Substituting the results of the previous into (17), we arrive at

$$
\begin{aligned}
\dot{V}_{k}\left(\bar{x}_{k}\right) \leq-(n-k & +1) \sum_{i=1}^{k-1} \xi_{i}^{2}+\left[\xi_{k}\right]^{2-r_{k+1} p_{k}} x_{k+1}^{* p_{k}} \\
& +\tilde{\kappa}_{k}\left(\bar{x}_{k}\right) \xi_{k}^{2}+\left[\xi_{k}\right]^{2-r_{k+1} p_{k}}\left(x_{k+1}^{p_{k}}-x_{k+1}^{* p_{k}}\right)+k \delta,
\end{aligned}
$$

where

$$
\tilde{\kappa}_{k}\left(\bar{x}_{k}\right)=a_{k}+b_{k}\left(\bar{x}_{k}\right)+c_{k}\left(\bar{x}_{k}\right)
$$

is a smooth positive function.

Now, it easy to see that the virtual controller

$$
x_{k+1}^{*}=-\beta_{k}^{r_{k+1}}\left(\bar{x}_{k}\right)\left[\xi_{k}\right]^{r_{k+1}},
$$

where $\beta_{k}\left(\bar{x}_{k}\right) \geq\left((n-k+1)+\tilde{\kappa}_{k}\left(\bar{x}_{k}\right)\right)^{1 / k_{k+1} p_{k}}$ is a smooth function, renders

$$
\dot{V}_{k}\left(x_{1}, \ldots, x_{k}\right) \leq-(n-k+1) \sum_{i=1}^{k} \xi_{i}^{2}+\left[\xi_{k}\right]^{2-r_{k+1} p_{k}}\left(x_{k+1}^{p_{k}}-x_{k+1}^{* p_{k}}\right)+k \delta .
$$

This completes the inductive step.

Using the inductive argument above, we can conclude that at the $n$-th step, there exists a continuous state feedback controller of the form

$$
u=x_{n+1}^{*}=-\beta_{n}^{r_{n+1}}\left(\bar{x}_{n}\right)\left[\xi_{n}\right]^{r_{n+1}}
$$

with the $C^{1}$, proper and positive definite Lyapunov function $V_{n}\left(x_{1}, x_{2} \ldots, x_{n}\right)$ constructed via the inductive procedure, we arrive at

$$
\dot{V}_{n}\left(x_{1}, \ldots, x_{n}\right) \leq-\sum_{i=1}^{n} \xi_{i}^{2}+n \delta .
$$

Noting that $\tau \in\left(-1 / \sum_{l=1}^{n} p_{1} \cdots p_{l-1}, 0\right)$ and $r_{k+1} p_{k}=r_{k}+\tau$, we have $0<r_{k+1} p_{k}<1$.

Moreover, recall that $V\left(x_{1}, \ldots, x_{n}\right)=\sum_{k=1}^{n} W_{k}\left(x_{1}, \ldots, x_{k}\right)$, where $W_{k}$ 's are defined in (13).

Then, it follows from Lemma5, we have

$$
W_{k}\left(\bar{x}_{k}\right) \leq\left|x_{k}-x_{k}^{*}\right|\left|\xi_{k}\right|^{2-r_{k+1} p_{k}} \leq 2^{1-r_{k}}\left|\xi_{k}\right|^{2-\tau} \leq 2\left(\left|\xi_{k}\right|^{2}\right)^{\sigma}
$$

So we have the following estimate:

$$
V_{n}\left(\bar{x}_{n}\right)=\sum_{k=1}^{n} W_{k}\left(\bar{x}_{k}\right) \leq 2 \sum_{k=1}^{n}\left|\xi_{k}\right|^{\sigma} .
$$

Let $\sigma=(2-\tau) / 2$. By $\tau \in\left(-1 / \sum_{l=1}^{n} p_{1} \cdots p_{l-1}, 0\right), 1 / \sigma \in(0,1)$. With (21) and (23) in mind, by Lemma4, it is not difficult to obtain that

$$
\dot{V}_{n}\left(\bar{x}_{n}\right) \leq-\left(V_{n}\left(\bar{x}_{n}\right) / 2\right)^{1 / \sigma}+n \delta
$$


It will show that the state $x(t)$ of closed-loop system (7) is well-defined on $[0,+\infty)$ and globally bounded. First, introduce the following set

$$
S:=\left\{x(t) \in R^{n} \mid V_{n}\left(\bar{x}_{n}\right) \geq 2(2 n \delta)^{\sigma}\right\},
$$

and let $x(t)$ be the trajectory of (7) with an initial state $x(0)$. If $x(t) \in S$, then it follows from (25) that

$$
\dot{V}_{n}\left(\bar{x}_{n}\right) \leq-\left(V_{n}\left(\bar{x}_{n}\right) / 2\right)^{1 / \sigma}+n \delta \leq-n \delta<0 .
$$

This implies that, as long as $x(t) \in S, V_{n}(x(t))$ is strictly decreasing with time $t$, and hence $x(t)$ must enter the complement set $R^{n}-S$ in a finite time $T \geq 0$ and stay there forever. Thus, the solution $x(t)$ of the system (7) is well-defined and globally bounded on $[0,+\infty)$. Next, it will be shown that

$$
\left|y(t)-y_{r}(t)\right|=\left|z_{1}(t)-y_{r}(t)\right|<\varepsilon, \quad \forall t \geq T>0 .
$$

This is also easily shown from (15), (24) and by tuning the parameter $\delta$ :

$$
\left|y(t)-y_{r}(t)\right|=\left|x_{1}(t)\right| \leq V_{n}(x(t)) \leq 2(2 n \delta)^{\sigma}<\varepsilon .
$$

Therefore, for any $\varepsilon>0$, there is globally practical output-tracking such that (27) holds. This completes the proof of Theorem 1.

\section{Conclusions}

In this paper, the global practical tracking problem has been studied for a class of highorder non-linear systems with more general uncertainties and presented a continuous state feedback output tracking controller for a class of high-order nonlinear systems under weaker condition. The controller guarantees that the states of the closed-loop system are globally bounded, while the tracking error can be bounded by any given positive number after a finite time. It should be noted that the proposed controller can only work well when the whole state vector is measurable. Therefore, a natural and more interesting problem is how to design output feedback output tracking controller for the systems studied in the paper if only partial state vector being measurable, which is now under our further investigation.

\section{Appendix}

Proof of Fact 1: Noting that $\tau \in\left(-1 / \sum_{l=1}^{n} p_{1} \cdots p_{l-1}, 0\right)$ and $r_{k} p_{k-1}=r_{k-1}+\tau$, we have $0<r_{k} p_{k-1}<1$. Using (11), it follows from Lemma5 that

$$
\begin{aligned}
\left|x_{k}^{p_{k-1}}-x_{k}^{*} p_{k-1}\right| & =\left|\left(x_{k}^{1 / r_{k}}\right)^{r_{k} p_{k-1}}-\left(x_{k}^{* 1 / r_{k}}\right)^{r_{k} p_{k-1}}\right| \\
& \leq 2^{1-r_{k} p_{k-1}}\left|\left[x_{k}\right]^{1 / r_{k}}-\left[x_{k}^{*}\right]^{1 / r_{k}}\right| r_{k}^{r_{k} p_{k-1}} \\
& =2^{1-r_{k} p_{k-1}}|\xi|^{r_{k} p_{k-1}} .
\end{aligned}
$$

By (A.1) and Lemma3, it can be obtained that 


$$
\begin{aligned}
{\left[\xi_{k-1}\right]^{2-r_{k} p_{k-1}}\left(x_{k}^{p_{k-1}}-x_{k}^{*} p_{k-1}\right) } & \leq 2^{1-r_{k} p_{k-1}}\left|\xi_{k-1}\right|^{2-r_{k} p_{k-1}}\left|\xi_{k}\right|^{r_{k} p_{k-1}} \\
& \leq \frac{1}{3} \xi_{k-1}^{2}+a_{k} \xi_{k}^{2}
\end{aligned}
$$

where $a_{k}>0$ is a constant.

Proof of Fact 2 : According to (11), Assumption 1, and Lemma2, it follows that

$$
\begin{aligned}
& \left|\phi_{k}\left(x_{1}+y_{r}, x_{2}, \ldots, x_{k}\right)\right| \\
& \quad \leq \psi_{k}\left(x_{1}+y_{r}, x_{2} \ldots, x_{k}\right)\left(\left|x_{1}+y_{r}\right|^{\left(r_{1}+\tau\right) / r_{1}}+\left|x_{2}\right|^{\left(r_{2}+\tau\right) / r_{2}}+\ldots+\left|x_{k}\right|^{\left(r_{k}+\tau\right) / r_{k}}\right) \\
& \quad \leq \tilde{\psi}_{k}\left(x_{1}, \ldots, x_{k}\right) \sum_{j=1}^{k}\left(\left|\xi_{j}\right|+\beta_{j-1}\left|\xi_{j-1}\right|\right)^{r_{k+1} p_{k}}+\tilde{\psi}_{k}\left(x_{1}, \ldots, x_{k}\right) M^{r_{k+1} p_{k}} \\
& \quad \leq 2^{1-r_{k+1} p_{k}} \tilde{\psi}_{k}\left(x_{1}, \ldots, x_{k}\right) \sum_{j=1}^{k}\left(\left|\xi_{j}\right|^{r_{k+1} p_{k}}+\beta_{j-1}^{r_{k+1} p_{k}}\left|\xi_{j-1}\right|^{r_{k+1} p_{k}}\right) \\
& \quad \leq \bar{\psi}_{k}\left(x_{1}, \ldots, x_{k}\right) \sum_{j=1}^{k}\left|\xi_{j}\right|^{r_{k+1} p_{k}}+\bar{\psi}_{k}\left(x_{1}, \ldots, x_{k}\right) M^{r_{k+1} p_{k}}
\end{aligned}
$$

where $\beta_{0}=0, \xi_{0}=0$ and $\bar{\psi}_{k}(\cdot)=2^{1-r_{k+1} p_{k}} \sum_{j=1}^{k}\left(1+\beta_{j-1}^{r_{k^{*}} p_{k}}\right) \tilde{\psi}_{k}(\cdot) \geq 0$ is a smooth function.

Using (A.3) and Lemmas3 and 5, we obtain,

$$
\begin{aligned}
& {\left[\xi_{k}\right]^{2-r_{k+1} p_{k}} \phi_{k}\left(x_{1}+y_{r}, x_{2}, \ldots, x_{k}\right)} \\
& \leq \bar{\psi}_{k}\left(x_{1}, \ldots, x_{k}\right) \sum_{j=1}^{k}\left|\xi_{k}\right|^{2-r_{k+1} p_{k}}\left|\xi_{j}\right|^{r_{k+1} p_{k}} \\
& \quad+\bar{\psi}_{k}\left(x_{1}, \ldots, x_{k}\right)\left|\xi_{k}\right|^{2-r_{k+1} p_{k}} M^{r_{k+1} p_{k}} \\
& \leq \frac{1}{3} \sum_{j=1}^{k-1} \xi_{j}^{2}+b_{k}\left(x_{1}, \ldots, x_{k}\right) \xi_{k}^{2}+\frac{1}{2} \delta
\end{aligned}
$$

where $b_{k}\left(x_{1}, \ldots, x_{k}\right)>0$ is a smooth function.

Proof of Fact 3 : Note that

$$
\left[x_{j+1}^{*}\right]^{1 / r_{j+1}}=-\beta_{j} \xi_{j}=-\sum_{j=1}^{k-1} B_{j}\left[x_{j}\right]^{1 / r_{j}},
$$

where $B_{j}=\beta_{k-1} \cdots \beta_{j}, j=1, \ldots, k-1$.

Using (A.5), after simple calculations, it is not hard to obtain that for $j=1, \ldots, k-1$,

$$
\frac{\partial\left[x_{k}^{*}\right]^{1 / r_{k}}}{\partial x_{j}}=-\sum_{j=1}^{k-1} \frac{\partial B_{l}}{\partial x_{j}}\left[x_{j}\right]^{1 / r_{j}}-\frac{1}{r_{j}} B_{j}\left|x_{j}\right|^{1 / r_{j}-1} .
$$

By (14), (15), (A.3), (A.6), and Lemmas2 and 5, we get 


$$
\begin{aligned}
\sum_{j=1}^{k-1} \frac{\partial W_{k}}{\partial x_{j}} \dot{x}_{j} & =\sum_{j=1}^{k-1} \frac{\partial W_{k}}{\partial x_{j}}\left(x_{j+1}^{p_{j}}+\psi_{j}(\cdot)\right) \\
& =-\left(2-r_{k+1} p_{k}\right) \int_{x_{k}^{*}}^{x_{k}}\left([s]^{1 / r_{k}}-\left[x_{k}^{*}\right]^{1 / r_{k}}\right)^{2-r_{k+1} p_{k}} d s \\
& \cdot \sum_{j=1}^{k-1} \frac{\partial\left[x_{k}^{* 1 / r_{k}}\right]}{\partial x_{j}}\left(x_{j+1}^{p_{j}}+\psi_{j}(\cdot)\right) \\
& \leq 2^{2-r_{k}}\left|\xi_{k}\right|^{1-\tau} \sum_{j=1}^{k-1}\left|\frac{\partial\left[\alpha_{k-1}^{1 / r_{k}}\right]}{\partial x_{j}}\right|\left(\left|x_{j+1}\right|^{p_{j}}+\left|\psi_{j}(\cdot)\right|\right) \\
& \leq \sum_{j=1}^{k-1} \bar{B}_{j}\left|\xi_{k}\right|^{1-\tau}\left|\xi_{j}+\beta_{j-1} \xi_{j-1}\right|^{1-r_{j}} \\
& \leq\left. 2^{2-r_{k}}\left|\xi_{k}\right|^{1-\tau} \sum_{j=1}^{k-1}\left|\sum_{l=1}^{k-1} \frac{\partial B_{l}}{\partial x_{j}}\right| x_{j}\right|^{+\frac{1}{r_{j}}} B_{j}\left|x_{j}\right|^{1 / r_{j}-1} \mid\left(\left|x_{j+1}\right|^{p_{j}}+\left|\psi_{j}(\cdot)\right|\right) \\
& \leq \sum_{j=1}^{k-1} \bar{B}_{j}\left|\xi_{k}\right|^{1-\tau}\left|x_{j}\right|^{1 / r_{j}-1}\left(\left|x_{j+1}\right|^{p_{j}}+\left|\psi_{j}(\cdot)\right|\right),
\end{aligned}
$$

where $\bar{B}_{j} \geq 0$ is a smooth function.

Noting that $r_{j+1} p_{j}=r_{j}+\tau$, by using Lemma3, we have

$$
\begin{aligned}
\sum_{j=1}^{k-1} \frac{\partial W_{k}}{\partial x_{j}} \dot{x}_{j} & =\sum_{j=1}^{k-1} \frac{\partial W_{k}}{\partial x_{j}}\left(x_{j+1}^{p_{j}}+\psi_{j}(\cdot)\right) \\
& \leq \frac{1}{3} \sum_{j=1}^{k-1} \xi_{j}^{2}+c_{k}(\cdot) \xi_{j}^{2}+\frac{1}{2} \delta,
\end{aligned}
$$

where $c_{k}(\cdot)$ is a smooth function.

\section{Acknowledgment}

This work was supported in part by JSPS KAKENHI Grant Number 26420428.

\section{References}

[1] A. Isidori and C. I. Byrnes, "Output regulation of nonlinear system”, IEEE Trans. on Automatic Control, vol. 35, (1990), pp. 31-140.

[2] C. I. Byrnes, F. D. Psicoli and A. Isidori, "Output Regulation of Uncertain Nonlinear Systems", Boston: Birkhäuser, (1997).

[3] C. J. Qian and W. Lin, "Practical output tracking of nonlinear systems with uncontrollable unstable linearization”, IEEE Trans. on Automatic Control, vol. 47, (2002), pp. 21-36.

[4] L. Marconi and A. Isidori, "Mixed internal model-based and feedforward control for robust tracking in nonlinear systems", Automatica, vol. 36, (2000), pp. 993-1000.

[5] W. Lin and R. Pongvuthithum, "Adaptive output tracking of inherently nonlinear systems with nonlinear parameterization", IEEE Trans. on Automatic Control, vol. 48, (2003), pp. 1737-1749.

[6] Z. Y. Sun and Y. G. Liu, "Adaptive practical output tracking control for high-order nonlinear uncertain systems”, Acta Automatica sinica, vol. 34, (2008), pp. 984-989.

[7] K. Alimhan and H. Inaba, "Practical output tracking by smooth output compensator for uncertain nonlinear systems with unstabilisable and undetectable linearization”, Int. Journal of Modelling, 
Identification and Control, vol. 5, (2008), pp. 1-13.

[8] K. Alimhan and H. Inaba, "Robust practical output tracking by output compensator for a class of uncertain inherently nonlinear systems", Int. Journal of Modeling, Identification and Control, vol. 4, (2008), pp. 304-314.

[9] W. P. Bi and J. F. Zhang, "Global practical tracking control for high-order nonlinear uncertain systems", Proc. of the Chinese Control and Decision Conference, (2010); Xuzhou, China.

[10] K. Alimhan, N. Otsuka, M. N. Kalimoldayev and A. A. Adamov, "Output tracking problem of uncertain nonlinear systems with high-order nonlinearities", Proc. of the 8th International Conference on Control and Automation, (2015); Jeju Island, Korea.

[11] A. Abdelkrim, C. Ghorbel and M. Benrejeb, "LMI-based tracking control for Takagi-Sugeno fuzzy model", Int. Journal of Control and Automation, (2010), pp. 21-36.

[12] W. Lin and C. J. Qian, "Adding one power integrator: a tool for global stabilization of high-order lowertriangular systems", Systems \& Control Letters, vol. 39, (2000), pp. 339-351.

[13] C. J. Qian and W. Lin, "Non-Lipschitz continuous stabilizers for nonlinear systems with uncontrollable unstable linearization", Systems \& Control Letters, vol. 42, (2001), pp. 185-200.

[14] C. J. Qian and W. Lin, "A continuous feedback approach to global strong stabilization of nonlinear systems", IEEE Trans. on Automatic Control, vol. 46, (2001), pp. 1061-1079.

[15] B. Yang and W. Lin, "Homogeneous observers, Iterative design, and global stabilization of high-order nonlinear systems by output feedback", IEEE Trans. on Automatic Control, vol. 49, (2004), pp. 10691080.

[16] B. Yang and W. Lin, "Robust output feedback stabilization of uncertain nonlinear systems with uncontrollable and unobservable linearization”, IEEE Trans. on Automatic Control, vol. 50, (2005), pp. 619-630.

[17] C. Qian and W. Lin, "Recursive observer design, homogeneous approximation, and nonsmooth output feedback stabilization of nonlinear systems", IEEE Trans. on Automatic Control, vol. 51, (2006), pp. 1457-1471.

[18] J. Polendo and C. Qian, "A generalized homogeneous domination approach for global stabilization of inherently non-linear systems via output feedback", Int. Journal of Robust Nonlinear Control, vol. 17, (2007), pp. 605-629.

[19] J. Polendo and C. Qian, "A universal method for robust stabilization of nonlinear systems: unification and extension of smooth and non-smooth approaches", Proc. of the American Control Conference, (2006); Minneapolis, MN, USA.

[20] X. Huang, W. Lin and B. Yang, "Finite-time stabilization in the large for uncertain nonlinear systems", Proc. of the American Control Conference, (2004); Boston, MA, USA.

[21] X. H. Zhang and X. J. Xie, "Global state feedback stabilization of nonlinear systems with high-order and low-order nonlinearitiesities”, Int. Journal of Control, vol. 87, (2014), pp. 642-652.

\section{Authors}

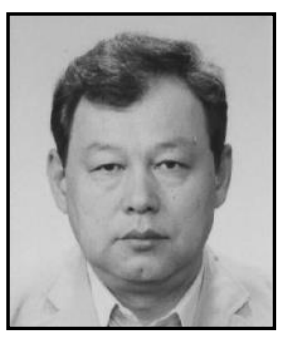

Keylan Alimhan, $\mathrm{He}$ received the B.S. degree in Mathematical Sciences in 1985 from Xinjiang University (XU), China, and M.S. degree in Information Sciences in 1998 from Tokyo Denki University (TDU), Japan and he finished his doctoral candidate course work in 2003 at TDU. In March 2009, he received the Doctor of Science degree in Mathematical Sciences from Graduate School of Science and Engineering of TDU. From 1985 to 1996, he served as an Assistant Professor in the Department of Mathematics, XU. From 2003 to 2004, he was a Research Associate in the Department of Information Sciences, TDU. From 2004 to present, he served as a Instructor, Assistant Professor and Research Fellow in School of Science and Engineering, TDU. Since September 2014, he has been with Faculty of Mechanics and Mathematics, Eurasian National University by named L.N.Gumilev as a Visiting Professor. His main research interests include nonlinear control theory, in particular, output feedback control of nonlinear systems and 
nonlinear robust control. Dr. Alimhan is a member of the Society of Instrument and Control Engineers of Japan.

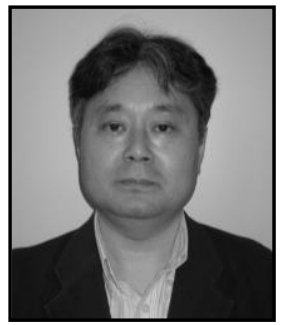

Naohisa Otsuka, He was born in Niigata, Japan. He received the B.S. and M.S. degrees, both in Mathematical Sciences, from Tokyo Denki University (TDU) in 1984 and 1986, respectively. In 1992, he received the Doctor of Science in Mathematical Sciences from Graduate School of Science and Engineering of TDU. From 1986 to 1992 he served as a Research Associate in the Department of Information Sciences, TDU. In April 1992, he moved to the Institute of Information Sciences and Electronics, University of Tsukuba as a Research Associate and served as an Assistant Professor of the same University from 1993 to September 2000. From October 2000 to September 2003 he served as an Associate Professor in the Department of Information Sciences, TDU. Since October 2003 he served as a Professor in the same department in TDU. Since April 2007 he has been with the division of Science in TDU as a Professor. His main research interests are in mathematical systems theory, in particular, geometric control theory, robust stability problems of uncertain systems and stability problems of switched linear systems. Dr. Otsuka is each member of the Society of Instrument and Control Engineers (SICE) of Japan, IEEE and SIAM. He is currently an Associate Editor of Journal of The Franklin Institute and an Editor of Mathematical Problems in Engineering.

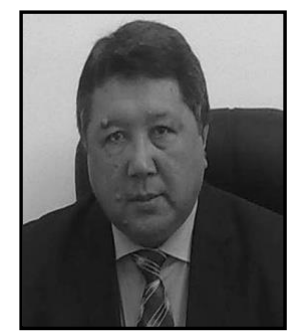

Kalimoldayev Maksat, He was born on June 18, 1957 in Almaty region, Kazakhstan. In 1975-1980 studied at the faculty of Mechanics and Applied mathematics of the Kazakh State University named after S.M.Kirov on the specialty Applied mathematics. From 1980 to 1982 he worked as an engineer of the laboratory of Mathematical methods of the Institute of Mathematics and Mechanics of the Academy of Sciences of the Kazakh SSR. Since 1982 he has started to work in the field of control theory. From that moment to the present the life and work of M. N. Kalimoldayev inextricably linked to Kazakh State University. Here he passed the way from Senior laboratory assistant, graduate student, senior lecturer assistant, associate Professor at the Control Theory Department to Professor of information systems Department of KazNU named after al-Farabi. M. N. Kalimoldayev is the author of over 120 research papers including 4 monographs and 5 study guides for universities and high school graduate, 1 certificate on the state registration of the intellectual property object, 2 certificates of the state registration of rights to the copyright object. Organizational skills of M.N. Kalimoldaev allowed him to coordinate scientific and teaching activities with administrative work. Since 2008 - general director of the Institute of Information and Computational Technologies of Science Committee of the Ministry of Education and Science. In February $28^{\text {th }}$ of 2013 he was elected as a corresponding member of National Academy of Sciences of Kazakhstan. 


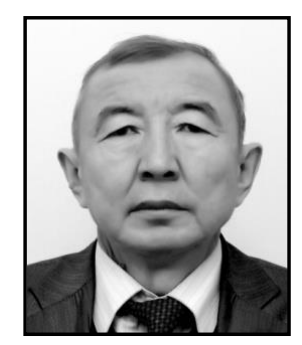

Abilmajin A. Adamov, He was born in 1955, December 10th, in Baschi of Almaty area, Kazakhstan. In 1977 he has received a Diploma of Higher Education in Mathematics studied in Kazakhstan State University named by S.M. Kirov, Alma-Ata, Kazakhstan. In 1983-1987 he had Scientific Research Training and Post -Graduate course studies at the Leningrad State University by name A.A. Zhdanov, Leningrad, Russia. In 1988 he has received a Diploma of Candidate of Physical and Mathematical Sciences (Ph.D.) in Leningrad State University by name A.A.Zhdanov, Leningrad, Russia. 1977-1981 - Department of exploitation the COMPUTER RCC CSD, senior engineer, chief of change, Almaty, Kazakhstan. 1981-1996 - Department of Calculus Mathematics of Kazakhstan State University by named S.M.Kirov, assistant, senior teacher, senior lecturer, assistant of the dean, Almaty, Kazakhstan. 1996-1998 - Faculty of all engineering disciplines of Technologies and Business Institute, Dean, Almaty, Kazakhstan. 1998-2000 - Faculty of natural - scientific disciplines Academy Civil Aircraft, Dean. 2000-2012Faculty «Computer facilities and information systems» of the Eurasian National University (ENU) by named L.N.Gumilev, senior lecturer, Professor. Since September 2013, he is the head of the Department of mathematics and computer modelling, Faculty of Mechanics and Mathematics, ENU. 
International Journal of Control and Automation Vol.9, No.1 (2016) 